\title{
Glucose uptake and growth of glucose-limited chemostat cultures of Aspergillus niger and a disruptant lacking MstA, a high-affinity glucose transporter
}

\section{Correspondence \\ Jens J. L. Iversen \\ jji@bmb.sdu.dk}

Received 12 December 2006

Revised 8 February 2007

Accepted 12 February 2007
Thomas R. Jørgensen, ${ }^{1}$ Patricia A. vanKuyk, ${ }^{2,3}$ Bjarne R. Poulsen, ${ }^{2} \dagger$ George J. G. Ruijter, ${ }^{2} \ddagger$ Jaap $\operatorname{Visser}^{2} \S$ and Jens J. L. Iversen ${ }^{1}$

\footnotetext{
${ }^{1}$ Department of Biochemistry and Molecular Biology, University of Southern Denmark, Campusvej 55, 5230 Odense M, Denmark

${ }^{2}$ Section Molecular Genetics of Industrial Microorganisms, Wageningen University, Dreijenlaan 2, 6703 HA Wageningen, The Netherlands

${ }^{3}$ Leiden University, Institute of Biology Leiden, Clusius Laboratory, Department of Fungal Genetics and Metabolomics, Wassenaarseweg 64, $2333 \mathrm{AL}$ Leiden, The Netherlands
}

This is a study of high-affinity glucose uptake in Aspergillus niger and the effect of disruption of a high-affinity monosaccharide-transporter gene, $m s t A$. The substrate saturation constant $\left(K_{\mathrm{s}}\right)$ of a reference strain was about $15 \mu \mathrm{M}$ in glucose-limited chemostat culture. Disruption of $m s t A$ resulted in a two- to fivefold reduction in affinity for glucose and led to expression of a low-affinity glucose transport gene, $m s t C$, at high dilution rate. The effect of $m s t A$ disruption was more subtle at low and intermediate dilution rates, pointing to some degree of functional redundancy in the high-affinity uptake system of $A$. niger. The $m s t A$ disruptant and a reference strain were cultivated in glucose-limited chemostat cultures at low, intermediate and high dilution rate $\left(D=0.07 \mathrm{~h}^{-1}\right.$, $0.14 \mathrm{~h}^{-1}$ and $0.20 \mathrm{~h}^{-1}$ ). Mycelium harvested from steady-state cultures was subjected to glucose uptake assays, and analysed for expression of $m s t A$ and two other transporter genes, $m s t C$ and $m s t F$. The capacity for glucose uptake $\left(v_{\max }\right)$ of both strains was significantly reduced at low dilution rate. The glucose uptake assays revealed complex uptake kinetics. This impeded accurate determination of maximum specific uptake rates $\left(v_{\max }\right)$ and apparent affinity constants $\left(K_{\mathrm{m}}^{\mathrm{app}}\right)$ at intermediate and high dilution rate. Two high-affinity glucose transporter genes, $m s t A$ and $m s t F$, were expressed at all three dilution rates in chemostat cultures, in contrast to batch culture, where only $m s t C$ was expressed. Expression patterns of the three transporter genes suggested differential regulation and functionality of their products.

\section{INTRODUCTION}

Soil-inhabiting filamentous fungi are adapted to an ecological niche in which they live on decaying organic matter in an environment characterized by scarcity of readily available nutrients. These fungi have an inherent ability to secrete enzymes that convert polymers into their monomeric constituents. This extracellular conversion of a biologically inert substrate into easily metabolized compounds necessitates the existence of an efficient uptake

tPresent address: Novozymes A/S, Hallas Allé 1, Building BD3.50.3, 4400 Kalundborg, Denmark.

$\ddagger$ Present address: Department of Clinical Genetics, Erasmus Medical Center, PO Box 1738, 3000 DR Rotterdam, The Netherlands.

§Present address: Fungal Genetics and Technology Consultancy, PO Box 396, 6700 AJ Wageningen, The Netherlands. mechanism to sustain growth in competition with other micro-organisms. Monod (1942) first reported that the specific growth rate of bacterial cultures was dependent on nutrient availability, best described by Michaelis-Menten enzyme kinetics.

Much research describes fungal growth as a result of uptake of monosaccharides, which are ubiquitous in ripe fruit, a substratum for many types of yeast, and the products of enzyme-catalysed degradation of carbohydrate polymers. Glucose has been the main focus as the substrate for glycolysis, the central metabolic pathway in most organisms.

Substrate saturation constants $\left(K_{\mathrm{s}}\right)$ have been determined, using chemostat cultivation, to relate growth to glucose availability. In the filamentous fungus Aspergillus oryzae, $K_{\mathrm{s}}$ for glucose is $30 \mu \mathrm{M}$ during glucose-limited growth in chemostat culture (Carlsen et al., 1996). In contrast, Larsen 
et al. (2004) reported a $K_{\mathrm{s}}$ for glucose of $19 \mathrm{mM}$ during growth of $A$. oryzae in batch cultures. $K_{\mathrm{s}}$ values determined for growth in both batch and chemostat cultures depend on substrate uptake, metabolism and growth. Many investigations, however, have focused on separation of the characterization of the uptake process from interfering effects of growth and metabolism by determination of initial glucose uptake rates of starved or washed cells (MacCabe et al., 2003; Mark \& Romano, 1971; Torres et al., 1996; vanKuyk et al., 2004; Walsh et al., 1994), isolated plasma membrane vesicles (Fuhrmann et al., 1989) or by description of uptake of non-metabolizable glucose analogues (Brown \& Romano, 1969; Moore \& Devadatham, 1979).

If substrate uptake obeys Michaelis-Menten kinetics, the process must be governed by a single rate-limiting reaction. Single affinity constants $\left(K_{\mathrm{m}}\right)$ have been determined for uptake of glucose by Aspergillus niger, $K_{\mathrm{m}}=150 \mu \mathrm{M}$ (Mischak et al., 1984), and Aspergillus nidulans, $K_{\mathrm{m}}=40$ $60 \mu \mathrm{M}$ (Brown \& Romano, 1969; Mark \& Romano, 1971). However, it was frequently observed that glucose uptake kinetics do not conform to a Michaelis-Menten description of uptake by a simple one-component system. In such cases deviations are often accounted for by involvement of additional transport systems. Thus, Torres et al. (1996) determined two $K_{\mathrm{m}}$ values for glucose uptake in A. niger. One, $K_{\mathrm{m}}=260 \mu \mathrm{M}$, represents a high-affinity transport system and the second, $K_{\mathrm{m}}=3.67 \mathrm{mM}$, a low-affinity system. Two transport systems, with $K_{\mathrm{m}}$ values of $16 \mu \mathrm{M}$ and $1.4 \mathrm{mM}$, respectively, have been described in $A$. nidulans (MacCabe et al., 2003). These results indicate that the number of identifiable uptake systems and their transport characteristics are highly dependent on experimental conditions. Glucose uptake in A. niger is reportedly mediated by a high-affinity and a low-affinity transport system. The latter is only detectable at high glucose concentrations (15\%) (Torres et al., 1996).

A wide array of putative sugar transport proteins in fungi has been described to date. They belong to the sugar porter (SP) family of the major facilitator superfamily (MFS), and are membrane-bound proteins containing 12 putative transmembrane regions. The proteins in this family function as uniporters or $\mathrm{H}^{+}$-symporters. They transport a variety of compounds, but many are specific for sugars (MFS is reviewed by Pao et al., 1998). The most detailed descriptions of hexose transporters in fungi are from research on Saccharomyces cerevisiae, in which at least 20 different proteins (the majority encoded by $h x t$ genes) are involved in transport of glucose; some of these transporters also have a broader specificity and mediate uptake of other hexoses as well, and some have been implicated in sensing and signalling (Wieczorke et al., 1999; the diversity and function of hexose transporters in S. cerevisiae are reviewed by Rolland et al., 2002). In A. nidulans there are at least 17 putative hexose transporters (Wei et al., 2004) and 20 have been identified in Candida albicans (Fan et al., 2002). The sheer number of transporters in one organism supports the idea of transport systems as products of differentially expressed transporter genes. The individual proteins would have specificity with respect to the type of substrate transported, and to the affinity and capacity of the transport process. Controlled and combined use of such proteins gives the organism flexibility in nutrient uptake, enabling it to adapt to various nutritional conditions.

Defined experimental conditions provide a basis for understanding mechanisms and processes involved in glucose uptake. Well-defined experimental growth conditions are only achievable through chemostat cultivation. Several studies have used this method to characterize glucose uptake of Candida utilis (Peinado et al., 1989; Postma et al., 1988, 1989a) and S. cerevisiae (Diderich et al., 1999; du Preez et al., 2000; Postma et al., 1989a, b). In the present study we used the well-defined conditions created by chemostat cultivation to describe high-affinity glucose uptake of $A$. niger. We determined glucose uptake kinetics of mycelium from the chemostat cultures in a separate uptake assay reactor and compared this to glucose uptake of chemostat cultures (in situ). Steady-state expression of three monosaccharide transporter $(m s t)$ genes was described by Northern blot analysis. In addition, we examined the effect of disrupting expression of $m s t A$, a high-affinity monosaccharide transporter involved in $\mathrm{H}^{+}$dependent glucose uptake (symport) (vanKuyk et al., 2004).

\section{METHODS}

Strains and inocula. Aspergillus niger NW325 [reference strain; $\triangle \operatorname{argB}$ goxC17 pyrA6 leuA1 nicA1 pIM2104 $\left.\left(\arg B^{+}\right)\right]$and NW326 ( $\triangle m s t A$ strain; $\triangle \arg B$ goxC17 pyrA6 leuA1 nicA1 $\triangle m s t A:: \arg B^{+}$) were used in this study. The strains were derived from $A$. niger $\mathrm{N} 400$ (CBS120.49) (vanKuyk et al., 2004). The conidial inocula for chemostat cultivation were obtained from cultures on complete medium agar plates (Pontecorvo, 1953) with $10 \mathrm{~g} g l u c o s e ~^{-1}$ as major carbon source. The medium was supplemented with $200 \mathrm{mg}$ leucine, $122 \mathrm{mg}$ uridine, $1 \mathrm{mg}$ nicotinic acid (niacin) and $1 \mathrm{ml}$ trace metal solution per litre medium; $\mathrm{pH}$ was adjusted to 6 with $\mathrm{NaOH}$. The trace metal solution contained, per litre: $10 \mathrm{~g}$ EDTA, $4.4 \mathrm{~g}$ $\mathrm{ZnSO}_{4} \cdot 7 \mathrm{H}_{2} \mathrm{O}, 1.01 \mathrm{~g} \mathrm{MnCl}_{2} \cdot 4 \mathrm{H}_{2} \mathrm{O}, 0.32 \mathrm{~g} \mathrm{CoCl}_{2} \cdot 6 \mathrm{H}_{2} \mathrm{O}, 0.315 \mathrm{~g}$ $\mathrm{CuSO}_{4} \cdot 5 \mathrm{H}_{2} \mathrm{O}, 0.22 \mathrm{~g}\left(\mathrm{NH}_{4}\right)_{6} \mathrm{Mo}_{7} \mathrm{O}_{24} \cdot 4 \mathrm{H}_{2} \mathrm{O}, 1.47 \mathrm{~g} \mathrm{CaCl}_{2} \cdot 2 \mathrm{H}_{2} \mathrm{O}$ and $1 \mathrm{~g} \mathrm{FeSO}_{4} \cdot 7 \mathrm{H}_{2} \mathrm{O}$ (modified from the composition given by Vishniac \& Santer, 1957). Leucine, uridine and nicotinic acid were added to complement auxotrophic requirements. One to two weeks before inoculation in the bioreactor, complete medium plates were inoculated with conidia from a stock culture (which had been cultivated on the same medium, and stored at $4{ }^{\circ} \mathrm{C}$ ). The plates were incubated at $30{ }^{\circ} \mathrm{C}$ for 5 days and then stored at $4{ }^{\circ} \mathrm{C}$. Conidia were harvested, and washed with a detergent solution containing $0.05 \%$

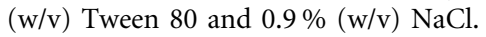

Chemostat cultivation. A stirred tank reactor, the 'Variomixing' bioreactor (Larsen et al., 2004), designed especially for cultivation of filamentous fungi, was used for chemostat cultivation. This bioreactor has unique features, which reduce attachment of biomass to bioreactor surfaces and concomitant wall growth. These qualities are essential for obtaining steady-state situations. Two major features of the bioreactor are intermittently rotating baffles and insertion of probes below the surface level of the culture, which reduce the surface 
area susceptible to wall growth. The baffles are used to change, depending on rotation speed, between turbulent and laminar mixing regimes. Turbulent mixing ensures good mixing and mass transfer qualities, whereas increased laminar flow near vessel surfaces prevents wall growth. The bioreactor was equipped with a Pt100 temperature sensor (PR Electronics), a 405-DPAS-SC-K8S/120 Combination pH sensor (Mettler Toledo) connected to a PHM 82 standard $\mathrm{pH}$ meter (Radiometer), a galvanic autoclavable oxygen electrode (Mackereth, 1964), and a Binos 100M gas analyser (Rosemount Analyticals). Temperature was $30{ }^{\circ} \mathrm{C}$, $\mathrm{pH}$ was 3 and the airflow rate was $11 \mathrm{~min}^{-1}$ (approx. $0.25 \mathrm{vvm}$ ). $\mathrm{pH}$ was maintained constant by titration with $2 \mathrm{M}$ $\mathrm{NaOH}$ delivered by a $101 \mathrm{~F} / 102 \mathrm{R}$ peristaltic pump (Watson-Marlow). The dissolved oxygen tension was always above $40 \%$ of air saturation at any time during continuous cultivation. Three weighing cells, SHBxR 9102 (Revere Transducers Europe), monitored culture mass. In chemostat mode a diaphragm pump (Hughes Pumps) supplied medium to the culture at constant flow rates, and constant culture mass was achieved through operation of a computer-regulated outlet situated in the bottom-plate of the bioreactor.

A 4.31 volume of minimal medium was inoculated with harvested conidia to give a concentration of $10^{9}$ conidia $1^{-1}$. The minimal medium contained, per litre: $4.5 \mathrm{~g} \mathrm{NH}_{4} \mathrm{Cl}, 1.5 \mathrm{~g} \mathrm{KH}_{2} \mathrm{PO}_{4}, 0.5 \mathrm{~g} \mathrm{KCl}$, $0.5 \mathrm{~g} \mathrm{MgSO}_{4} \cdot 7 \mathrm{H}_{2} \mathrm{O}, 1 \mathrm{ml}$ trace metal solution, $200 \mathrm{mg}$ leucine, 122 $\mathrm{mg}$ uridine and $1 \mathrm{mg}$ nicotinic acid. Polypropylene glycol (PPG 2000, Fluka Chemika; $0.01 \%$, w/v) was added to reduce foaming. The concentration of the final-cell-density-limiting substrate, glucose, was $7.5 \mathrm{~g} \mathrm{l}^{-1}$. In the initial batch culture $0.003 \%(\mathrm{w} / \mathrm{v})$ yeast extract was added to the medium, prior to inoculation, to stimulate germination of conidia. After two-thirds of the glucose had been consumed, batch cultivation was switched to chemostat mode. Steady state was defined by constant biomass and glucose concentrations, base titration rate, $\mathrm{CO}_{2}$ evolution rate and $\mathrm{O}_{2}$ consumption rate after at least three retention times. Steady-state cultures provided mycelium for glucose uptake assays in a separate uptake assay reactor, and Northern blot analysis. Mycelium from mid-exponential batch cultures was also analysed for mst expression. Samples of up to $100 \mathrm{ml}$ were retrieved within $1 \mathrm{~s}$, through a sampling device without dead-space. The sampling device was slightly modified from that described by Iversen (1981). For the assessment of biomass homogeneity in cultures and assays, it is important to note that the morphology was filamentous with some minor mycelial aggregates, but without pellets.

Glucose uptake assays. Uptake assays were conducted in a conventional stirred-tank bioreactor (glucose uptake assay bioreactor) to allow successive fast sampling from a well-mixed system. The dimensions of the bioreactor were: height, $130 \mathrm{~mm}$; diameter, $100 \mathrm{~mm}$. Mixing efficiency, expressed as the characteristic mixing time $\left(t_{\text {mix }}\right.$ or $\tau$ ), and time to achieve $90 \%$ homogeneity were 1 and $2 \mathrm{~s}$, respectively, when determined by the method of Poulsen \& Iversen (1997). The broth was sufficiently aerated to avoid oxygen limitation during the experiments, as the dissolved oxygen tension was always above $40 \%$ of air saturation.

To begin each uptake assay, $100 \mathrm{ml}$ steady-state culture broth was transferred from the chemostat culture to $400 \mathrm{ml}$ preconditioned medium in the uptake assay bioreactor. The sampling of $100 \mathrm{ml}$ broth from the chemostat culture and subsequent transfer to the uptake assay bioreactor was accomplished in approximately $5 \mathrm{~s}$. For each dilution rate, at steady state, four different uptake assays were performed with initial glucose concentrations of 48, 80, 160 and $240 \mu \mathrm{M}$, respectively. The preconditioned medium was otherwise identical to the medium used in chemostat cultivation; however, it did not contain antifoam agent. The temperature was $30{ }^{\circ} \mathrm{C}$ and $\mathrm{pH}$ was 3. The glucose uptake assays lasted 5-6 min. Samples were taken every 15-30 s, depending on the initial glucose concentration. A sampling device similar to the one used in the 'Variomixing' bioreactor (Iversen, 1981) was used to sample broth in less than $1 \mathrm{~s}$. The mycelium was separated from the broth by filtration on a cotton filter within $1 \mathrm{~s}$ of sampling. A special filtration device was constructed for this purpose (Fig. 1). It consisted of a $10 \mathrm{ml}$ syringe connected with a short piece of silicone tubing $(6 / 4 \mathrm{~mm})$ to a $5 \mathrm{ml}$ plastic micropipette tip, which contained a plug of cotton wool. The filtrate was frozen in liquid nitrogen. At the end of each assay a sample was taken to determine biomass concentration.

Determination of glucose and biomass. Precipitate was removed from culture filtrate from both chemostat and uptake assay samples by centrifugation, and the supernatant was used for further analysis. Glucose concentration was determined enzymically using a method described by Bergmeyer et al. (1974). The method was slightly modified by using $250 \mathrm{mM}$ triethanolamine as buffer. Dry weight biomass concentration was determined by separation of mycelium from a known volume of broth on a Whatman glass microfibre filter. The mycelium was washed with demineralized water, frozen in liquid nitrogen and freeze-dried until constant weight.

Determination of specific glucose uptake rates in glucose uptake assays and estimation of glucose uptake parameters. Time and glucose concentration were averaged to reduce noise prior to determination of the specific glucose uptake rates $(v)$. Adjacent glucose concentrations were averaged; the slope between adjacent averages was calculated and divided by the biomass concentration as shown in Fig. 2. Results from four assays with different initial glucose concentrations for each strain were used to characterize glucose uptake in accordance with the best fit of Michaelis-Menten kinetics (Fig. 3):

$v=v_{\max } s\left(s+K_{\mathrm{m}}^{\mathrm{app}}\right)^{-1}$

where $v_{\max }$ is the maximum specific glucose uptake rate $\left(\mu \mathrm{mol} \mathrm{g} \mathrm{dw}^{-1}\right.$ $\mathrm{s}^{-1}$ ) and $K_{\mathrm{m}}^{\mathrm{app}}$ is the apparent affinity constant for glucose uptake $(\mu \mathrm{M})$. (Symbols used in this paper are listed in Table 1.)

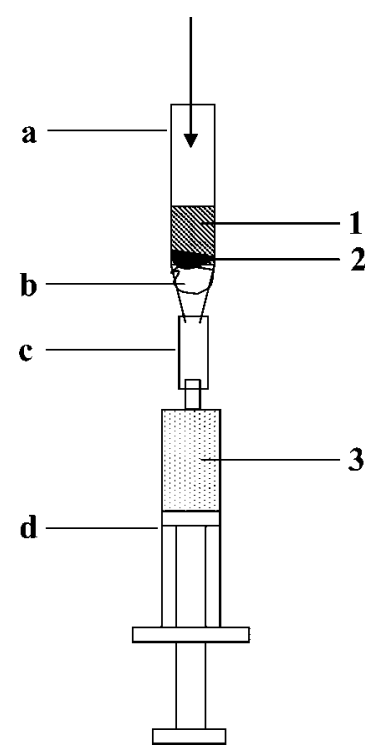

Fig. 1. Filtration of culture broth in glucose uptake assays. Fivemillilitre micropipette tip (a), cotton wool (b), silicone tubing (c), $10 \mathrm{ml}$ syringe (d), culture broth (1), mycelium (2) and filtrate (3). The arrow indicates sample flow. 


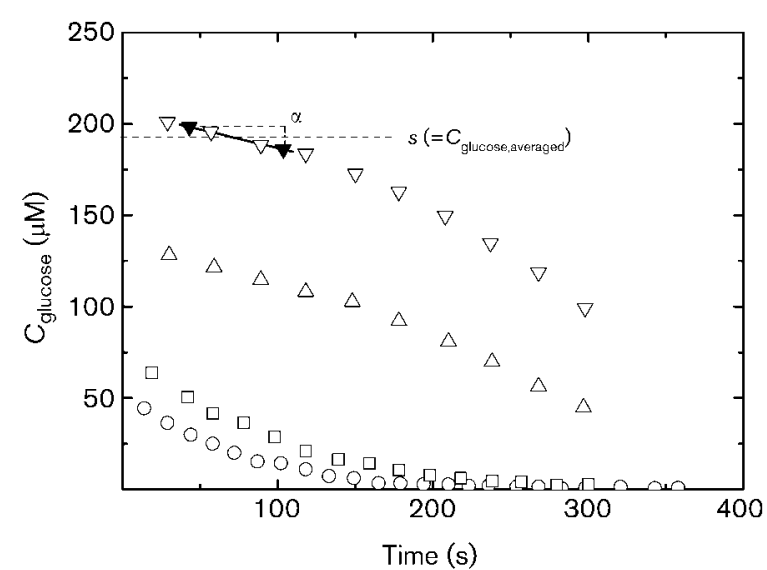

Fig. 2. Determination of glucose uptake. Example of glucose uptake by mycelium obtained from a steady-state culture (NW325 at $\left.D=0.20 \mathrm{~h}^{-1}\right)$. Each symbol represents an assay with initial glucose concentration of $48 \mu \mathrm{M}(\bigcirc), 80 \mu \mathrm{M}(\square), 160 \mu \mathrm{M}(\triangle)$ or $240 \mu \mathrm{M}(\nabla)$. Specific uptake rates $(v)$ used for parameter determination (equation 1 and Fig. 3) were calculated from mean values as illustrated here $\left(v=-\alpha \mathrm{C}_{\text {biomass }}{ }^{-1}\right)$ and explained in Methods. Filled symbols are mean values derived from pairs of adjacent substrate concentrations; $s$ is the average glucose concentration correlated with a calculated specific uptake rate.

Before fitting of equation 1 the results (filled symbols in Fig. 3) that apparently deviated from saturation kinetics were omitted. Data points of individual uptake assays were omitted if they represented an increase in specific uptake rate $(v)$ with decreasing substrate concentrations $(s)$. This assumes that uptake inhibition or metabolic bottlenecks affected uptake at higher glucose concentrations (see Discussion).

Calculation of substrate saturation constants and metabolic quotients for comparison of glucose uptake in chemostat cultures and glucose uptake assays. The substrate saturation constant $\left(K_{\mathrm{s}}\right)$ for glucose of steady-state chemostat cultures was determined from the following expression, which was derived from chemostat theory (Monod, 1950):

$K_{s}=\tilde{s}\left(\mu_{\max } D^{-1}-1\right)$

where $\tilde{s}$ is the steady-state glucose concentration $(\mu \mathrm{M}), \mu_{\max }$ is the maximum specific growth rate $\left(\mathrm{h}^{-1}\right)$ determined in batch cultures and $D$ is the dilution rate $\left(\mathrm{h}^{-1}\right)(=$ specific growth rate, $\mu$, at steady state).

The in situ specific glucose uptake rate of chemostat cultures is equal to the metabolic quotient $(q)$ for glucose at steady state and was calculated from the expression

$q=\mu\left(s_{\mathrm{r}}-\tilde{s}\right) \tilde{x}^{-1}$

where $\mu$ is the specific growth rate $\left(\mathrm{h}^{-1}\right)$ (=D at steady state), $s_{\mathrm{r}}$ is the concentration of glucose in the influent medium $(\mu \mathrm{M})$ and $\tilde{x}$ is the steady-state biomass concentration $\left(\mathrm{g}_{\mathrm{dw}} \mathrm{l}^{-1}\right)$.

Glucose uptake determined in the glucose uptake assays was compared to glucose uptake of steady-state chemostat cultures $(q)$ by calculation of metabolic quotients $\left(q_{\text {up }}\right)$ for glucose based on results of glucose uptake assays from the expression $q_{\mathrm{up}}=v_{\max } \tilde{s}\left(\tilde{s}+K_{\mathrm{m}}^{\mathrm{app}}\right)^{-1}$

This method was previously used by Postma et al. (1989b) to compare in situ glucose uptake of S. cerevisiae chemostat cultures (in situ) with glucose uptake kinetics determined in glucose uptake assays with cells obtained from the chemostat cultures.

Northern blot analysis of mst expression. Culture broth from batch and steady-state chemostat cultures was filtered on sintered glass in a funnel and the retained mycelium washed with ice-cold phosphate buffer $\left(25 \mathrm{mM} \mathrm{KH} \mathrm{KH}_{4}\right.$ and $\left.50 \mathrm{mM} \mathrm{K}_{2} \mathrm{HPO}_{4}\right) \mathrm{pH} 7$. Excess liquid was removed by suction and by pressing the mycelium with a paper towel. The partially dried mycelium was rapidly frozen in liquid nitrogen. The entire duration of sampling and preparation was less than $20 \mathrm{~s}$. The samples were stored at $-80{ }^{\circ} \mathrm{C}$ until analysis. RNA was extracted from mycelial samples, and prepared for Northern blot analysis according to previously described methods (vanKuyk et al., 2004). Probes used were: $1.95 \mathrm{~kb}$ EcoRI-XhoI fragment from mstA cDNA clone pIM4903 (sequence accession no. AY081845); a 725 bp PstI-SstI internal fragment of $m s t C$ (sequence accession no. AY081847); and an EcoRI-Xbal fragment of approx. $1.7 \mathrm{~kb}$ of pIM4930 corresponding to the full-length $m s t F \mathrm{cDNA}$. A $0.7 \mathrm{~kb} E c o$ RI fragment from the gene encoding the $18 \mathrm{~S}$ rRNA subunit (Melchers et al., 1994) was used as an RNA loading control.

\section{RESULTS}

\section{Steady-state glucose uptake and growth of chemostat cultures}

Two strains, A. niger NW325 (reference) and NW326 $(\triangle m s t A)$, were cultivated at low, intermediate and high dilution rates $(D): 0.07,0.14$ and $0.20 \mathrm{~h}^{-1}$. The dilution rates corresponded, respectively, to $0.24,0.48$ and 0.69 of the maximum specific growth rate $\left(\mu_{\max }\right)$, which was $0.29 \mathrm{~h}^{-1}$ for both strains in batch culture. Steady-state concentrations of biomass $(\tilde{x})$ and glucose $(\tilde{s})$ and yield constants $(Y)$ are listed in Table 2.

Glucose-limited growth of the reference strain, at intermediate and high dilution rates $\left(0.14 \mathrm{~h}^{-1}\right.$ and $\left.0.20 \mathrm{~h}^{-1}\right)$, obeyed chemostat theory (Monod, 1950), which assumes a constant maximum specific growth rate $\left(\mu_{\max }\right)$ and substrate saturation constant $\left(K_{\mathrm{s}}\right)$. The $K_{\mathrm{s}}$ at these dilution rates was approximately $15 \mu \mathrm{M}$. The culture growing at low dilution rate $\left(0.07 \mathrm{~h}^{-1}\right)$ had a four- to fivefold higher $K_{\mathrm{s}}$.

Disruption of $m s t A$ expression resulted in higher glucose concentrations at steady state (Table 2). The change of phenotype as a result of disruption was clearly demonstrated at high dilution rate $\left(0.20 \mathrm{~h}^{-1}\right)$, where steady-state glucose concentration $(\tilde{s})$ and substrate saturation constant $\left(K_{\mathrm{s}}\right)$ were five times higher than the corresponding values of the reference strain. In addition, it was observed that cultures of the mstA disruptant reached steady state after a longer period compared with cultures of the reference strain (i.e. it took longer to reach constant glucose concentration). In $m s t A$ disruptant cultures, at intermediate and high dilution rates $\left(0.14 \mathrm{~h}^{-1}\right.$ and $\left.0.20 \mathrm{~h}^{-1}\right)$, steady states were established five and four retention times $\left(D^{-1}\right)$ 

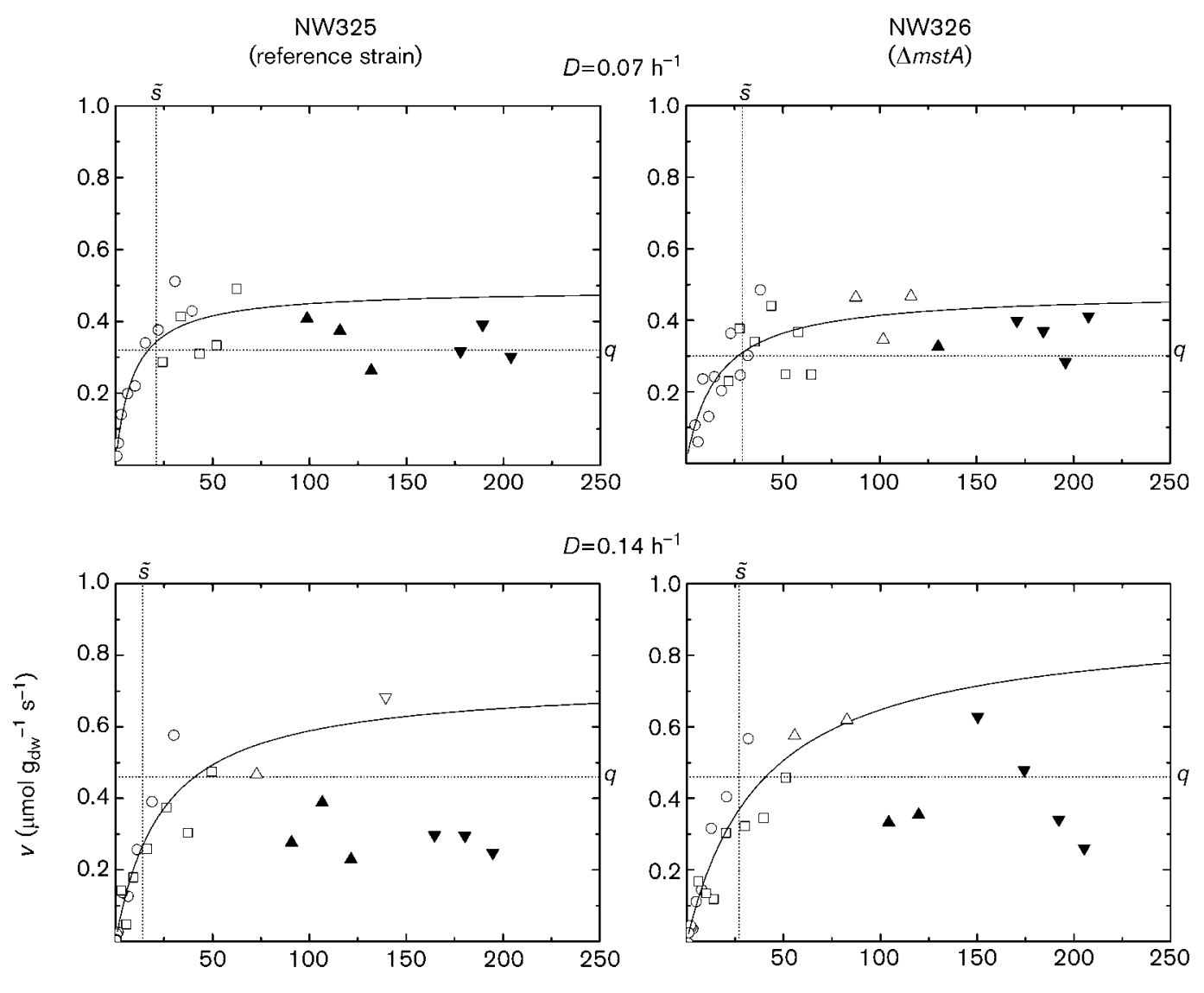

$D=0.20 \mathrm{~h}^{-1}$

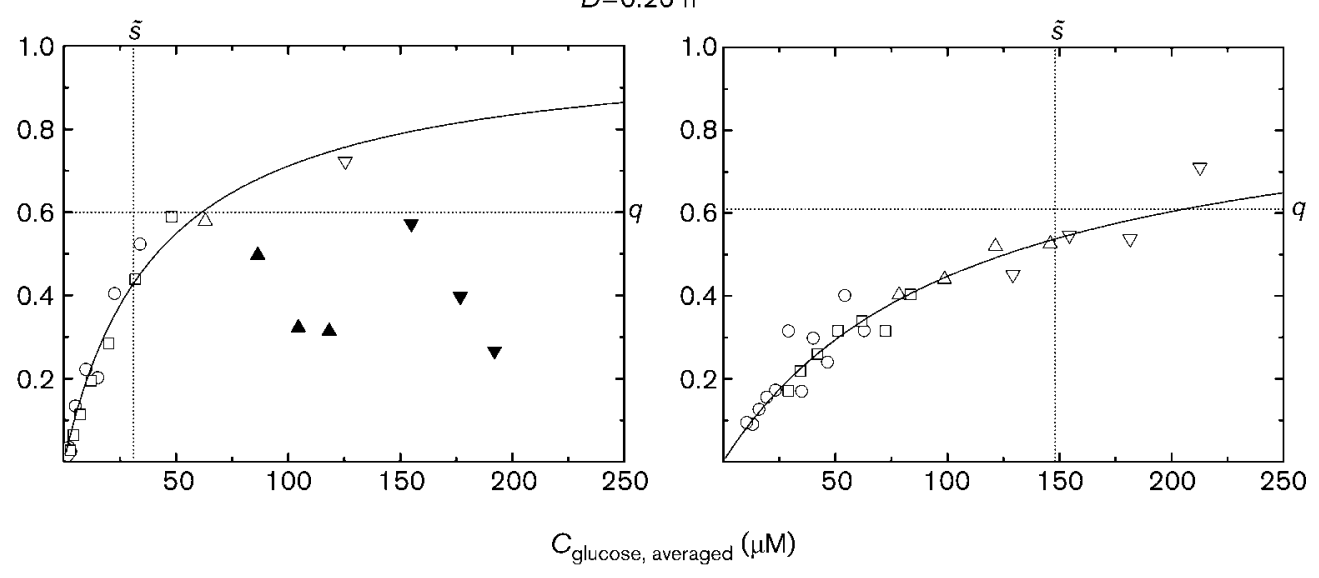

Fig. 3. Glucose uptake described by Michaelis-Menten kinetics. Glucose uptake kinetics of NW325 (reference strain) and NW326 ( $\Delta m s t A)$ mycelium, obtained from steady state at three dilution rates, $0.07 \mathrm{~h}^{-1}, 0.14 \mathrm{~h}^{-1}$ and $0.20 \mathrm{~h}^{-1}(0.24,0.48$ and 0.69 of $\left.\mu_{\text {max }}\right)$, in the glucose uptake assay bioreactor. Specific uptake rates $(v)$ as a function of glucose concentration in uptake assays with four different initial glucose concentrations: $48 \mu \mathrm{M}(\bigcirc), 80 \mu \mathrm{M}(\square), 160 \mu \mathrm{M}(\triangle)$ and $240 \mu \mathrm{M}(\nabla) . v_{\max }$ and $K_{\mathrm{m}}^{\text {app }}$ $( \pm \mathrm{SD})$ are listed in Table 2, as determined from a fit of equation 1 (solid curve) to $v$ vs $s$ ( $\mathrm{C}_{\text {glucose, averaged }}$ ). Filled symbols $(\boldsymbol{\bullet}, \mathbf{\square}$, $\boldsymbol{\Delta}, \boldsymbol{\nabla})$ represent omitted results (see Methods). $q$ and $\widetilde{s}$ are indicated by dotted lines in each figure for comparison of steadystate kinetics of glucose uptake of chemostat cultures with kinetics in the uptake assays.

after start of continuous cultivation, whereas steady states were established within three retention times in cultures of the reference strain. The mstA disruptant retained sig- nificant capacity of high-affinity glucose uptake both in chemostat cultures and in uptake assays on mycelium from the chemostat cultures. 
Table 1. Symbols

\begin{tabular}{|c|c|}
\hline Symbol & Quantity and units \\
\hline$D$ & Dilution rate $\left(\mathrm{h}^{-1}\right)$ \\
\hline$K_{\mathrm{s}}$ & Substrate saturation constant $(\mu \mathrm{M})$ \\
\hline$K_{\mathrm{m}}$ & Michaelis/affinity constant $(\mu \mathrm{M})$ \\
\hline$K_{\mathrm{m}}^{\mathrm{app}}$ & Apparent Michaelis/affinity constant $(\mu \mathrm{M})$ \\
\hline$\mu$ & Specific growth rate $\left(\mathrm{h}^{-1}\right)$ \\
\hline$\mu_{\max }$ & Maximum specific growth rate $\left(\mathrm{h}^{-1}\right)$ \\
\hline$q$ & Metabolic quotient $\left(\mu \mathrm{mol} \mathrm{g}_{\mathrm{dw}}{ }^{-1} \mathrm{~s}^{-1}\right)$ \\
\hline$q_{\max }$ & Maximum metabolic quotient $\left(\mu \mathrm{mol} \mathrm{g}_{\mathrm{dw}}{ }^{-1} \mathrm{~s}^{-1}\right)$ \\
\hline$q_{\text {up }}$ & $\begin{array}{l}\text { Metabolic quotient calculated from glucose uptake assay } \\
\text { and chemostat results }\left(\mu \mathrm{mol} \mathrm{g}_{\mathrm{dw}}{ }^{-1} \mathrm{~s}^{-1}\right)\end{array}$ \\
\hline$s$ & Substrate concentration $(\mu \mathrm{M})$ \\
\hline$\tilde{s}$ & Steady-state concentration $(\mu \mathrm{M})$ \\
\hline$s_{\mathrm{r}}$ & $\begin{array}{l}\text { Medium concentration of the final-cell-density-limiting } \\
\text { substrate }(\mu \mathrm{M})\end{array}$ \\
\hline$v$ & Specific uptake rate $\left(\mu \mathrm{mol} \mathrm{g}_{\mathrm{dw}}{ }^{-1} \mathrm{~s}^{-1}\right)$ \\
\hline$v_{\max }$ & Maximum specific uptake rate $\left(\mu \mathrm{mol} \mathrm{g}_{\mathrm{dw}}{ }^{-1} \mathrm{~s}^{-1}\right)$ \\
\hline Y & Yield constant $\left(\mathrm{g}_{\mathrm{dw}} \mathrm{g}_{\text {glucose }}{ }^{-1}\right)$ \\
\hline$x$ & Dry weight biomass concentration $\left(\mathrm{g}_{\mathrm{dw}} \mathrm{l}^{-1}\right)$ \\
\hline$\tilde{x}$ & Steady-state dry weight biomass concentration $\left(\mathrm{g}_{\mathrm{dw}} \mathrm{l}^{-1}\right)$ \\
\hline
\end{tabular}

\section{Non-steady-state glucose uptake kinetics in the uptake assay bioreactor}

Results of the uptake assays are shown in Fig. 3. The capacity of glucose uptake, expressed as the maximum specific glucose uptake rate $\left(v_{\max }\right)$, and apparent $K_{\mathrm{m}}\left(K_{\mathrm{m}}^{\mathrm{app}}\right)$ of reference strain mycelium and $m s t A$ disruptant mycelium obtained from three dilution rates at steady state are shown in Table 2.

Generally, the maximum specific glucose uptake rate $\left(v_{\max }\right)$ increased with increasing dilution rate for both strains. However, there was no significant difference in the uptake capacities $\left(v_{\max }\right)$ of mstA disruptant mycelium from intermediate and high dilution rates $\left(0.14 \mathrm{~h}^{-1}\right.$ and $0.20 \mathrm{~h}^{-1}$ ). The mstA disruptant had reduced affinity (higher $K_{\mathrm{m}}^{\mathrm{app}}$ ) for glucose uptake in comparison to the reference strain. The effect of $m s t A$ disruption was most pronounced in mycelia obtained from the high dilution rate $\left(0.20 \mathrm{~h}^{-1}\right)$, where a two- to threefold reduction in affinity was observed.

Deviations from Michaelis-Menten kinetics were observed in the glucose uptake assays (Figs 2 and 3). The specific uptake rate $(v)$ increased just after glucose addition, when the initial concentration of glucose was high $\left(C_{\text {glucose }} 160\right.$ and $240 \mu \mathrm{M}$ ), even though the glucose concentration $(s)$ decreased. For comparison, the medium flow of the chemostat was stopped, at steady state, creating a situation comparable to the conditions in the uptake assays. An aliquot of glucose was then added to the culture to give an initial concentration of about $500 \mu \mathrm{M}$. This also resulted in glucose uptake characterized by a low initial specific uptake rate $(v)$, which increased even though the extracellular glucose concentration decreased (results not shown). Thus, the phenomenon was not an artefact of the uptake assays in the glucose uptake assay bioreactor. The deviations were apparent in five out of six of the combined glucose uptake assay results in Fig. 3, and especially when the mycelium was obtained from intermediate and high dilution rates $\left(0.14 \mathrm{~h}^{-1}\right.$ and $\left.0.20 \mathrm{~h}^{-1}\right)$. Glucose uptake of the mstA disruptant, sampled at the high dilution rate $\left(0.20 \mathrm{~h}^{-1}\right)$, was an exception.

The steady-state glucose concentrations $(\tilde{s})$ and metabolic quotients for glucose $(q)$ are indicated in Fig. 3 in order to compare the kinetics determined in the uptake assays with in situ glucose uptake of the steady-state cultures. Fig. 4 shows the steady-state metabolic quotients $(q)$ and metabolic quotients for glucose calculated from glucose uptake parameters $\left(q_{\text {up }}\right)$. The chemostat results $(\tilde{s}$ and $q)$ indicate that glucose uptake and growth of chemostat

Table 2. Chemostat cultivation and glucose uptake assay for reference strain (NW325) and $m s t A \operatorname{disruptant}(\mathrm{NW} 326)$

Steady-state concentrations of glucose $(\tilde{s})$ and dry weight biomass $(\tilde{x})$. Numbers are means \pm SD, based on a minimum of three samples from each steady state. Yield constant, $Y$, was calculated from $\tilde{x}\left(s_{\mathrm{r}}-\tilde{s}\right)^{-1}$, where $s_{\mathrm{r}}$ is concentration of final cell-density-limiting substrate, glucose, in the growth medium. The substrate saturation constant, $K_{s}$, for glucose was calculated from equation 2 using $\mu_{\max }$ determined in batch culture $\left(0.29 \mathrm{~h}^{-1}\right)$ and tabulated values of $\tilde{s}$ and D. $K_{\mathrm{m}}^{\text {app }}$ and maximum specific uptake rate $\left(v_{\max }\right)$ were determined in glucose uptake assays by best fit of equation 1 (Fig. 3). Error of regression ( $95 \%$ confidence) is given $( \pm)$ for $K_{\mathrm{m}}^{\text {app }}$ and $v_{\max }$.

\begin{tabular}{|c|c|c|c|c|c|c|c|c|}
\hline \multirow[t]{2}{*}{$D\left(h^{-1}\right)$} & \multirow[t]{2}{*}{$D \mu_{\max }^{-1}$} & \multirow[t]{2}{*}{ Strain } & \multicolumn{4}{|c|}{ Chemostat culture } & \multicolumn{2}{|c|}{ Glucose uptake assay } \\
\hline & & & $\widetilde{\boldsymbol{s}}(\boldsymbol{\mu M})$ & $\tilde{x}\left(g_{d w} l^{-1}\right)$ & $Y\left(g_{d w} g_{\text {glucose }}{ }^{-1}\right)$ & $K_{\mathrm{s}}(\mu \mathrm{M})$ & $K_{\mathbf{m}}^{\mathrm{app}}(\mu \mathrm{M})$ & $v_{\max }\left(\mu \mathrm{mol} \mathrm{g}_{\mathrm{dw}}{ }^{-1} \mathrm{~s}^{-1}\right)$ \\
\hline \multirow[t]{2}{*}{0.07} & 0.24 & NW325 & $21 \pm 1$ & $2.55 \pm 0.02$ & $0.34 \pm 0.00$ & $67 \pm 2^{*}$ & $9 \pm 4$ & $0.49 \pm 0.06$ \\
\hline & & NW326 & $29 \pm 3^{*}$ & $2.69 \pm 0.04$ & $0.36 \pm 0.01$ & $91 \pm 8$ & $16 \pm 7$ & $0.48 \pm 0.06$ \\
\hline \multirow[t]{2}{*}{0.14} & 0.48 & NW325 & $14 \pm 1$ & $3.74 \pm 0.06^{\star}$ & $0.50 \pm 0.01^{*}$ & $15 \pm 1 \dagger$ & $24 \pm 7$ & $0.73 \pm 0.09$ \\
\hline & & NW326 & $27 \pm 2^{*}$ & $3.53 \pm 0.03$ & $0.47 \pm 0.00$ & $29 \pm 2$ & $39 \pm 14$ & $0.90 \pm 0.17$ \\
\hline \multirow[t]{2}{*}{0.20} & 0.69 & NW325 & $31 \pm 3^{*}$ & $3.87 \pm 0.04$ & $0.52 \pm 0.01^{*}$ & $14 \pm 1 \dagger$ & $42 \pm 7$ & $1.01 \pm 0.08$ \\
\hline & & NW326 & $148 \pm 2$ & $3.78 \pm 0.02^{*}$ & $0.51 \pm 0.00^{*}$ & $67 \pm 1^{*}$ & $108 \pm 19$ & $0.93 \pm 0.09$ \\
\hline
\end{tabular}

${ }^{*} \dagger$ Denote numbers in each column that are not significantly different from each other. Sample means were compared in two-tailed $t$-test; significance level: $P=0.05$. 


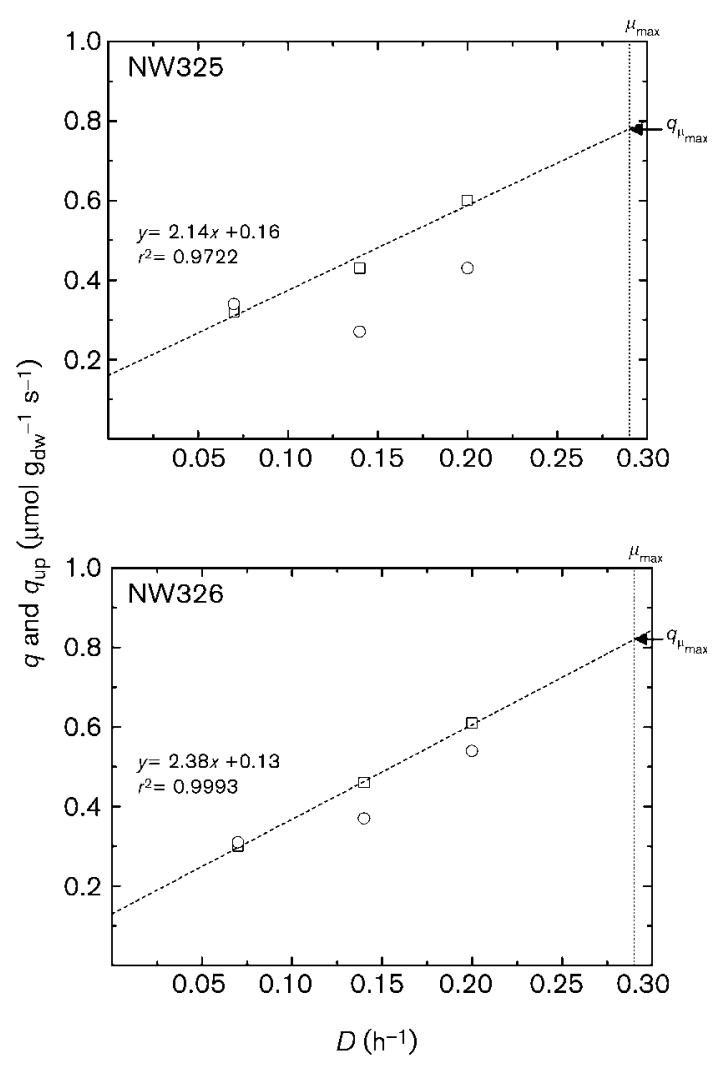

Fig. 4. Metabolic quotient for glucose at steady state $(q)$ and metabolic quotient for glucose calculated from glucose uptake parameters $\left(q_{\mathrm{up}}\right)$ vs dilution rate $(D)$ for NW325 (reference strain) and NW326 $(\Delta m s t A)$. The $q$ values $(\square)$ were calculated from equation 3 . The linear fit (dashed line) describes $q$ as a function of $D$ (= $\mu$ at steady state) according to the concept of a true growth yield and maintenance coefficient (Pirt, 1975). Arrows indicate a hypothetical $q_{\max }$ value, when $D=\mu_{\max }\left(=0.29 \mathrm{~h}^{-1}\right)$. The $q_{\text {up }}$ values $(\bigcirc)$ were calculated from equation 4.

cultures at low dilution rate $\left(0.07 \mathrm{~h}^{-1}\right)$ are governed by the same rate-limiting reaction as glucose uptake in the corresponding uptake assays (Figs 3 and 4). However, the metabolic quotients for glucose calculated from glucose uptake parameters $\left(q_{\text {up }}\right)$ of mycelium from intermediate and high dilution rates $\left(0.14 \mathrm{~h}^{-1}\right.$ and $\left.0.20 \mathrm{~h}^{-1}\right)$ were consistently lower than the steady-state metabolic quotients $(q)$. The results of individual pulse experiments shown in Fig. 3 suggest that the maximum specific uptake rates $\left(v_{\max }\right)$ have been underestimated and/or $K_{\mathrm{m}}^{\mathrm{app}}$ overestimated at these two dilution rates. From Fig. 4 it appears that the uptake parameters determined in the uptake assay reactor for the $m s t A$ distruptant described glucose uptake at steady state in the chemostat $(q)$ more accurately than those determined for the reference strain.

The metabolic quotient $\left(q_{\max }\right)$ at maximum specific growth rate $\left(\mu_{\max }\right)$ was approximately $0.8 \mu \mathrm{mol} \mathrm{g} \mathrm{dw}^{-1} \mathrm{~s}^{-1}$, when determined by extrapolation as shown in Fig. 4. The extrapolated intercept on the ordinate axis ( $y$-axis, $D=0$,
Fig. 4) represents the maintenance requirement (Pirt, 1975), which explains the low yield $(Y)$ and low steadystate biomass concentration (Table 2 ) at low dilution rate $\left(D=0.07 \mathrm{~h}^{-1}\right)$.

\section{Differential expression of genes encoding putative monosaccharide transporters}

Differential patterns of mst expression were observed under different growth conditions (Fig. 5).

Transcripts of $m s t A$ were detected in steady-state samples from all three dilution rates $\left(0.07,0.14\right.$ and $\left.0.20 \mathrm{~h}^{-1}\right)$. mst $A$ was not transcribed in detectable levels during batch cultivation, and transcription was not detected in cultures of the mstA disruptant, confirming the inability of this strain to express the $m s t A$ gene. In the reference strain $m s t A$ was expressed at the highest level at intermediate dilution rate $\left(0.14 \mathrm{~h}^{-1}\right)$. Probes for transcripts of two putative monosaccharide transporter genes, $m s t C$ and $m s t F$, were included in the Northern blot analysis. These genes were chosen as experimental results had shown that the proteins they encode are both able to transport D-glucose (P. A. vanKuyk, unpublished). The expression pattern of $m s t F$ is similar to that of $m s t A$, i.e. expression at low glucose concentrations, suggesting that it may also be a highaffinity transporter. Expression of $m s t C$ in the reference strain was not detectable in chemostat culture, but high during batch cultivation. The mstA disruptant differed from the reference strain in that $m s t C$ was transcribed at high dilution rate $\left(0.20 \mathrm{~h}^{-1}\right)$.

\section{DISCUSSION}

In this study we investigated glucose uptake of $A$. niger grown in chemostat cultures (in situ). Glucose uptake assays in a separate uptake assay reactor were used to characterize glucose uptake kinetics of mycelium obtained from steady state at different specific growth rates. Glucose uptake kinetics in the uptake assays was then compared with in situ glucose uptake $(q)$ by calculation of metabolic quotients based on glucose uptake assay results $\left(q_{\mathrm{up}}\right)$. We also studied steady-state expression of a high-affinity glucose transporter gene, $m s t A$, and two putative transporter genes, and the effect of disruption of mstA.

\section{High-affinity glucose uptake}

The substrate saturation constants $\left(K_{\mathrm{s}} 14-15 \mu \mathrm{M}\right)$ of the reference strain, determined at intermediate and high dilution rates $\left(0.14 \mathrm{~h}^{-1}\right.$ and $\left.0.20 \mathrm{~h}^{-1}\right)$, are $10-17$ times lower than the apparent affinity constants $\left(K_{\mathrm{m}}^{\mathrm{app}}\right)$ previously reported for high-affinity glucose uptake in A. niger (Torres et al., 1996; Mischak et al., 1984). The $K_{\mathrm{m}}^{\mathrm{app}}$ of $9 \pm 4 \mu \mathrm{M}$, determined in the glucose uptake assays on samples taken at low dilution rate $\left(0.07 \mathrm{~h}^{-1}\right)$, is similar to the values of the substrate saturation constant $\left(K_{\mathrm{s}}\right)$ at intermediate and high dilution rates $\left(0.14 \mathrm{~h}^{-1}\right.$ and 


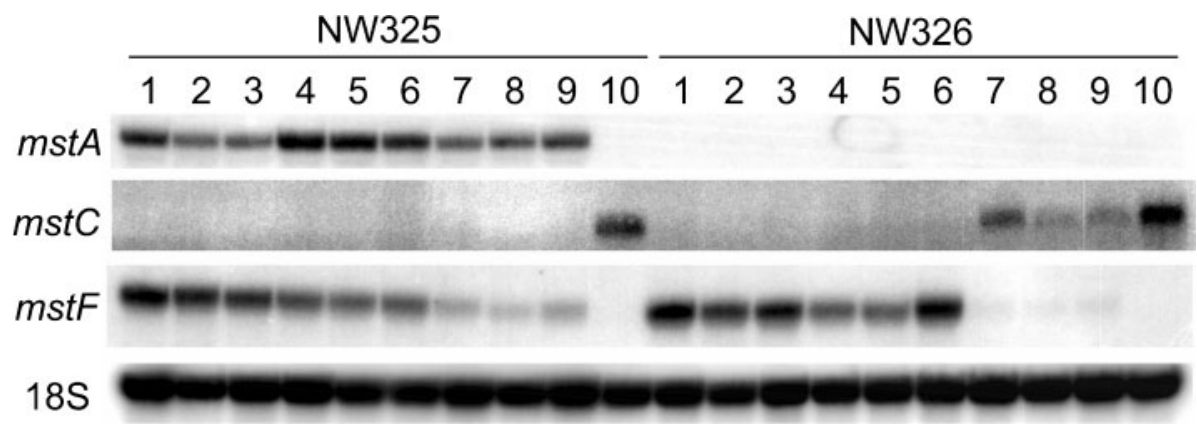

Fig. 5. Northern blot analysis of $m s t$ expression. Total RNA of NW325 (reference strain) and NW326 ( $\Delta m s t A)$ cultivated in batch and chemostat cultures was probed for expression of $m s t A, m s t C$ and $m s t F$. Lanes 1-3, triplicate samples from $D=0.07 \mathrm{~h}^{-1}$; lanes $4-6, D=0.14 \mathrm{~h}^{-1}$; lanes $7-9, \mathrm{D}=0.20 \mathrm{~h}^{-1}$; lane 10 , batch culture in exponential growth phase $\left(\mu_{\max }=0.29 \mathrm{~h}^{-1}\right)$. Steady-state glucose concentrations are shown in Table 2. Glucose concentrations in batch cultures were $24 \mathrm{mM}(0.44 \%, w / v)$ and $21 \mathrm{mM}(0.37 \%, w / v)$ for NW325 and NW326, respectively, when samples were taken for Northern blot analysis (lane 10). $18 \mathrm{~S}$ is a loading control.

$0.20 \mathrm{~h}^{-1}$ ) and, based on evaluation of the metabolic quotient calculated from glucose uptake assay results $\left(q_{\mathrm{up}}\right)$, it appears to reflect the true in situ affinity for glucose uptake at the low dilution rate $\left(0.07 \mathrm{~h}^{-1}\right)$. The most likely explanation for the high $K_{\mathrm{s}}$ value at low dilution rate $\left(0.07 \mathrm{~h}^{-1}\right)$ is discussed below.

The steady-state glucose concentrations are approximately equal to the affinity values of the mycelium (Table 2). This indicates that affinity $\left(K_{\mathrm{m}}\right)$ and uptake capacity $\left(v_{\max }\right)$ are adapted to ensure efficient regulation of metabolism at low substrate concentrations, since small changes in substrate concentration around the affinity concentration will lead to a significant change in uptake rate and metabolic fluxes.

\section{Reduced capacity of glucose uptake at low specific growth rate}

Steady-state glucose concentrations at low dilution rate $\left(0.07 \mathrm{~h}^{-1}\right)$, and hence the calculated saturation constants $\left(K_{\mathrm{s}}\right)$, were higher than predicted from chemostat theory for cultures of both reference strain and $m s t A$ disruptant. It seems that both reduced yield (i.e. low steady-state concentration of glucose-consuming biomass) and reduced uptake capacity $\left(v_{\max }\right)$ contribute to this deviation, rather than the reduction in affinity (high $K_{\mathrm{s}}$ values) shown in Table 2. This is inferred from the precise description of in situ glucose uptake by the uptake parameters determined in the glucose uptake assays, i.e. the steady-state metabolic quotient $(q)$ was equal to the metabolic quotient calculated from glucose uptake assay results $\left(q_{\mathrm{up}}\right)$. The $K_{\mathrm{s}}$ values listed in Table 2 were determined assuming that $\mu_{\max }$ is constant, independent of dilution rate. However, the maximum specific uptake rate $\left(v_{\max }\right)$, determined at low dilution rate $\left(0.07 \mathrm{~h}^{-1}\right)$ in the glucose uptake assays, was only about $60 \%$ of the specific uptake rate necessary to sustain a specific growth rate approximating the maximum, while the $K_{\mathrm{m}}^{\mathrm{app}}$ was similar to the $K_{\mathrm{s}}$ values calculated for the higher dilution rates $\left(0.14 \mathrm{~h}^{-1}\right.$ and $\left.0.20 \mathrm{~h}^{-1}\right)$ (see Table 2 and Fig. 4). In glucose-limited chemostat cultures of $A$. oryzae at low dilution rates $\left(D=0.025 \mathrm{~h}^{-1}\right.$ and $\left.0.10 \mathrm{~h}^{-1}\right)$, corresponding to 0.09 and 0.37 of the maximum specific growth rate $\left(\mu_{\max }\right)$, respectively, Carlsen et al. (1996) found that the maximum specific uptake rates $\left(v_{\max }\right)$ of glucose were lower than the $v_{\max }$ determined in batch culture. The lower capacity of glucose uptake $\left(v_{\max }\right)$ correlated with a lower content of stable RNA $\left(D<0.10 \mathrm{~h}^{-1}\right)$, and hence a reduced capacity of the translation machinery. If the capacity of glucose uptake depends on the amount of protein or energy allocated to uptake processes, it would follow that the maximum specific glucose uptake rate $\left(v_{\max }\right)$ of a culture changes according to the availability of the substrate and energy source, glucose.

A culture may contain both viable and non-viable biomass. The maximum specific glucose uptake rate $\left(v_{\max }\right)$ of both strains would be expected to decrease after the dilution rate was lowered (to $0.07 \mathrm{~h}^{-1}$ ), if the viability of the culture decreased. Autolysis is observed at low dilution rates in glucose-limited chemostat cultures of $A$. nidulans (Bainbridge et al., 1971). In addition, Agger et al. (1998) showed that the proportion of active biomass (containing organelles) in chemostat cultures of $A$. oryzae decreases with decreasing dilution rates. Thus the reduction of the maximum specific glucose uptake rate $\left(v_{\max }\right)$ may be a general response to starvation at low dilution rates, caused by a general reduction of metabolic capacities and/or an increasing amount of non-active biomass.

\section{Glucose uptake affected by more than one rate- limiting reaction}

It is a recurring phenomenon (Diderich et al., 1999; du Preez et al., 2000; Postma et al., 1989b) that specific glucose uptake rates or metabolic quotients for glucose calculated from parameters determined in glucose uptake assays $\left(q_{\text {up }}\right)$ 
are significantly lower than the in situ rates or metabolic quotients $(q)$, which are based on chemostat results (Fig. 4). The results presented in Fig. 3 may offer an explanation for these observations. The specific glucose uptake rates $(v)$ determined in the uptake assays were based on at least 10 consecutive samples from the uptake assay reactor; this allowed a temporal resolution of uptake, and the discovery of kinetics that did not conform to a Michaelis-Menten type model. The glucose uptake rate increased during the first 2-3 min in spite of decreasing glucose concentration. We observed the same when adding a pulse of glucose to glucose-limited chemostat cultures. Carlsen et al. (1996) observed a similar phenomenon, after adding pulses of glucose to glucose-limited chemostat cultures of A. oryzae at steady state, and suggested that the lag reflected constraints set by the capacity of the catabolic machinery (uptake system, glycolysis, TCA and oxidative phosphorylation) at steady state as an adaptation to starvation.

The translocation process per se is not the single ratelimiting step of glucose uptake in filamentous fungi. The results presented here, and other observations, indicate that metabolic reactions after translocation affect the rate of glucose uptake. In some studies $v_{\max }$ was the same for both high- and low-affinity transport systems, and for uptake of hexoses transported by different systems (Mark \& Romano, 1971; Scarborough, 1970b; Walsh et al., 1994). In Neurospora crassa it was shown that the maximum uptake rate was similar to the capacity of disrupted mycelium to phosphorylate glucose (Scarborough, 1970a). Glucoserepressed mycelium of Coprinus cinereus with low-affinity uptake was able to transport glucose with high affinity upon starvation (derepression) without de novo protein synthesis. This demonstrates that the proteins needed for high-affinity uptake were present in glucose-repressed mycelium (Moore \& Devadatham, 1979). Translocation and phosphorylation of glucose in aspergilli are two separate processes (Brown \& Romano, 1969). In a situation where the former process is faster than the latter, glucose will accumulate inside the cell, which leads to a reduction of the concentration gradient over the membrane. Description of glucose uptake affected by multiple metabolic bottlenecks or catabolite inhibition would require use of a segregated model, which includes such phenomena. Construction of a segregated model requires further studies, since the exact mechanisms behind the observed complexity of glucose uptake are insufficiently characterized.

\section{Growth-rate-dependent mst expression in chemostat culture}

Catabolite repression may explain the lack of $m s t A$ expression during batch culture, where the fungus grows at maximum specific growth rate $\left(\mu_{\max }\right)$ and glucose uptake is saturated. The promoter of $m s t A$ includes motifs that imply regulation of expression by the carbon catabolite repressor, CreA (vanKuyk et al., 2004). CreA mediates carbon catabolite repression and affects high-affinity uptake of glucose in A. nidulans (MacCabe et al., 2003). CreA has a function similar to the glucose repressor protein Miglp, which is involved in regulation of expression of the hxt genes in S. cerevisiae (reviewed by Rolland et al., 2002). The low level of $m s t F$ expression at high dilution rate $\left(0.20 \mathrm{~h}^{-1}\right)$ could have been caused by a tighter regulation in response to varying degrees of repression. Although the difference in extracellular glucose concentration (Table 2) at the three dilution rates seemed small, the changes in metabolic quotient $(q)$ for glucose, and hence metabolic fluxes, were significant (Fig. 4). In an extensive study of glucose uptake and $h x t$ expression in chemostat cultures of S. cerevisiae, Diderich et al. (1999) found differential hxt expression at different dilution rates. These authors concluded that expression patterns of the transporter genes correlated well with uptake parameters determined in separate uptake assays on cells from individual steady states, and the deduced function of single transport proteins (high/low affinity).

\section{Disruption of $\boldsymbol{m s t A}$}

vanKuyk et al. (2004) demonstrated that expression of $A$. niger $m s t A$ in a $S$. cerevisiae strain, deficient in hexose uptake by deletion of several hxt genes, results in highaffinity uptake of glucose, mannose and xylose and moderate-affinity uptake of fructose. The apparent $K_{\mathrm{m}}$ for glucose uptake mediated by the ectopic MstA was $25 \pm 10 \mu \mathrm{M}$. From this description of MstA function, we expected that disruption of $m s t A$ might have an effect on high-affinity glucose uptake in $A$. niger.

The maximum specific growth rate $\left(\mu_{\max }\right)$ of the mstA disruptant in batch culture was identical to that of the reference strain, and unaffected by the disruption of $m s t A$. This is expected, since $m s t A$ was not expressed by the reference strain in batch culture (Fig. 5).

Translocation of glucose in the glucose uptake assays was apparently less affected by putative downstream metabolic bottlenecks in the $m s t A$ disruptant compared to the reference strain (Fig. 4). Glucose uptake of $m s t A$ disruptant mycelium sampled at high dilution rate $\left(0.20 \mathrm{~h}^{-1}\right)$ appeared to obey Michaelis-Menten kinetics, unlike glucose uptake of reference strain mycelium obtained at the same dilution rate (Fig. 3). The reduced affinity of the $m s t A$ disruptant may have resulted in lower initial uptake rates and thus lower metabolic fluxes.

We found a two- to threefold reduction in apparent affinity for glucose, when the mstA disruptant was compared with the reference strain at high dilution rate $\left(0.20 \mathrm{~h}^{-1}\right)$. The $K_{\mathrm{m}}^{\mathrm{app}}$ of the $m s t A$ disruptant at this dilution rate also differed significantly from the affinities determined at the lower dilution rates $\left(0.07 \mathrm{~h}^{-1}\right.$ and $\left.0.14 \mathrm{~h}^{-1}\right)$. However, the $m s t A$ disruptant still retained capability of high-affinity glucose uptake. Loss of MstA function at high dilution rate $\left(0.20 \mathrm{~h}^{-1}\right)$ may have been partially complemented by 
upregulation of other glucose transporters, for example $\mathrm{MstC}$, which is not expressed at this dilution rate in the reference strain (Fig. 5). Expression of $m s t F$ is also affected by disruption of mstA in samples from high dilution rate $\left(0.20 \mathrm{~h}^{-1}\right)$. The expression results suggest that $m s t F$ encodes a high-affinity glucose transporter and that $m s t C$ encodes a transporter with lower affinity, normally expressed at higher extracellular glucose concentrations/ specific growth rates. These functional implications were supported experimentally by the functional analysis of $m s t F$ - and $m s t C$-encoded proteins in S. cerevisiae (P. A. vanKuyk, unpublished) and the $K_{\mathrm{m}}^{\mathrm{app}}$ values determined in the present study. Lack of the MstA protein leads to reduction of affinity/capacity for glucose uptake by mycelium cultivated at high dilution rate $\left(0.20 \mathrm{~h}^{-1}\right)$. The fungus responds by altering glucose transporter expression; $m s t F$ is downregulated and $m s t C$ upregulated. At a molecular level it is most likely that the carbon catabolite repressor, CreA, plays a negative role in this modulation. A positively acting regulator, which acts on the promoters of the glucose transporter genes, has not been identified yet. As the three transporters constitute only a fraction of the total number of sugar transporters expected to be encoded by the A. niger genome it is difficult to reach any definitive conclusions with respect to functional complementation.

In conclusion, the A. niger reference strain grew in glucoselimited chemostat culture with a substrate saturation constant $\left(K_{s}\right)$ of about $15 \mu \mathrm{M}$. The capacity of glucose uptake $\left(v_{\max }\right)$ was significantly reduced at low dilution rate $\left(0.07 \mathrm{~h}^{-1}\right)$. Determination of maximum specific uptake rates $\left(v_{\max }\right)$ and apparent affinity constants $\left(K_{\mathrm{m}}^{\mathrm{app}}\right)$ were affected by complex uptake kinetics, especially apparent at intermediate and high dilution rate $\left(0.14 \mathrm{~h}^{-1}\right.$ and $\left.0.20 \mathrm{~h}^{-1}\right)$. Two high-affinity glucose transporter genes, $m s t A$ and $m s t F$, were expressed at all three dilution rates, but not in batch culture, where only $m s t C$ was expressed. This suggested differential regulation and functionality of their products. Disruption of $m s t A$ resulted in a two- to fivefold reduction in affinity for glucose and led to expression of $m s t C$ at high dilution rate. Disruption of $m s t A$ had little effect at low and intermediate dilution rates, which indicated functional redundancy in the high-affinity uptake system of $A$. niger.

\section{ACKNOWLEDGEMENTS}

The authors would like to acknowledge Erling Knudsen for expert technical assistance and Sumi Wang for developing the filtration method used in the glucose uptake assay.

\section{REFERENCES}

Agger, T., Spohr, A. B., Carlsen, M. \& Nielsen, J. (1998). Growth and product formation of Aspergillus oryzae during submerged cultivations: verification of a morphologically structured model using fluorescent probes. Biotechnol Bioeng 57, 321-329.
Bainbridge, B. W., Bull, A. T., Pirt, S. J., Rowley, B. I. \& Trinci, A. P. J. (1971). Biochemical and structural changes in non-growing, maintained and autolysing cultures of Aspergillus nidulans. Trans Br Mycol Soc 56, 371-385.

Bergmeyer, H. U., Bernt, E., Schmidt, F. \& Stork, H. (1974). DGlucose determination with hexokinase and glucose-6-phosphate dehydrogenase. In Methods of Enzymatic Analysis 3, pp. 1196-1201. Edited by H. U. Bergmeyer. New York: Academic Press.

Brown, C. E. \& Romano, A. H. (1969). Evidence against necessary phosphorylation during hexose transport in Aspergillus nidulans. $J$ Bacteriol 100, 1198-1203.

Carlsen, M., Nielsen, J. B. \& Villadsen, J. (1996). Growth and $\alpha$ amylase production by Aspergillus oryzae during continuous cultivations. J Biotechnol 45, 81-93.

Diderich, J. A., Schepper, M., van Hoek, P., Luttik, A. H., van Dijken, J. P., Pronk, J. T., Klaassen, P., Boelens, H. F. M., de Mattos, M. J. T. \& other authors (1999). Glucose uptake kinetics and transcription of hxt genes in chemostat cultures of Saccharomyces cerevisiae. J Biol Chem 274, 15350-15359.

du Preez, J. C., de Kock, S. H., Kilian, S. G. \& Litthauer, D. (2000). The relationship between transport kinetics and glucose uptake by Saccharomyces cerevisiae in aerobic chemostat cultures. Antonie Van Leeuwenhoek 77, 379-388.

Fan, J., Chaturvedi, V. \& Shen, S. (2002). Identification and phylogenetic analysis of a glucose transporter family from the human pathogenic yeast Candida albicans. J Mol Evol 55, 336-346.

Fuhrmann, G. F., Völker, B., Sander, S. \& Potthast, M. (1989). Kinetic analysis and simulation of glucose transport in plasma membrane vesicles of glucose-repressed and derepressed Saccharomyces cerevisiae cells. Experientia 45, 1018-1023.

Iversen, J. J. L. (1981). A rapid sampling valve with minimal dead space for laboratory scale fermenters. Biotechnol Bioeng 23, 437-440.

Larsen, B., Rask Poulsen, B., Eriksen, N. T. \& Lønsmann Iversen, J. J. (2004). Homogeneous batch cultures of Aspergillus oryzae by elimination of wall growth in the Variomixing bioreactor. Appl Microbiol Biotechnol 64, 192-198.

MacCabe, A. P., Miró, P., Ventura, L. \& Ramón, D. (2003). Glucose uptake in germinating Aspergillus nidulans conidia: involvement of the creA and sorA genes. Microbiology 149, 2129-2136.

Mackereth, F. J. H. (1964). An improved galvanic cell for determination of oxygen concentrations in fluids. J Sci Instrum 41, 38-41.

Mark, C. G. \& Romano, A. H. (1971). Properties of the hexose transport systems of Aspergillus nidulans. Biochim Biophys Acta 249, 216-226.

Melchers, W. J. G., Verweij, P. E., van den Hurk, P., van Belkum, A., de Pauw, B. E., Hoogkamp-Korstanje, A. A. \& Meis, J. F. G. M. (1994). General primer-mediated PCR for detection of Aspergillus species. $J$ Clin Microbiol 32, 1710-1717.

Mischak, H., Kubicek, C. P. \& Röhr, M. (1984). Citrate inhibition of glucose uptake in Aspergillus niger. Biotechnol Lett 6, 425-430.

Monod, J. (1942). Recherches sur la Croissance des Cultures Bactériennes. Paris: Hermann (1958).

Monod, J. (1950). La technique de culture continue: théorie et applications. Ann Inst Pasteur 79, 390-410.

Moore, D. \& Devadatham, M. S. (1979). Sugar transport in Coprinus cinereus. Biochim Biophys Acta 550, 515-526.

Pao, S. S., Paulsen, I. T. \& Saier, M. H. (1998). Major facilitator superfamily. Microbiol Mol Biol Rev 62, 1-34.

Peinado, J. M., Cameira-Dos-Santos, P. J. \& Loureiro-Días, M. C. (1989). Regulation of glucose transport in Candida utilis. J Gen Microbiol 135, 195-201. 
Pirt, S. J. (1975). Principles of Microbe and Cell Cultivation. Oxford: Blackwell Scientific Publications.

Pontecorvo, G. (1953). The genetics of Aspergillus nidulans. In Advances in Genetics, vol. 5, pp. 141-238. Edited by M. Demerec. New York: Academic Press.

Postma, E., Scheffers, W. A. \& van Dijken, J. P. (1988). Adaptation of the kinetics of glucose transport to environmental conditions in the yeast Candida utilis CBS 621: a continuous-culture study. J Gen Microbiol 134, 1109-1116.

Postma, E., Kuiper, A., Tomasouw, W. F., Scheffers, A. \& van Dijken, J. P. (1989a). Competition for glucose between the yeasts Saccharomyces cerevisiae and Candida utilis. Appl Environ Microbiol 55, 3214-3220.

Postma, E., Scheffers, W. A. \& van Dijken, J. P. (1989b). Kinetics of growth and glucose transport in glucose-limited chemostat cultures of Saccharomyces cerevisiae CBS 8066. Yeast 5, 159-165.

Poulsen, B. R. \& Iversen, J. J. L. (1997). Mixing determinations in reactor vessels using linear buffers. Chem Eng Sci 52, 979-984.

Rolland, F., Winderickx, J. \& Thevelein, J. M. (2002). Glucose-sensing and -signalling mechanisms in yeast. FEMS Yeast Res 2, 183-201.

Scarborough, G. A. (1970a). Sugar transport in Neurospora crassa. J Biol Chem 245, 1694-1698.

Scarborough, G. A. (1970b). Sugar transport in Neurospora crassa. II. A second glucose transport system. J Biol Chem 245, 3985-3987.
Torres, N. V., Riol-Cimas, J. M., Wolschek, M. \& Kubicek, C. P. (1996). Glucose transport by Aspergillus niger: the low-affinity carrier is only formed during growth on high glucose concentrations. Appl Microbiol Biotechnol 44, 790-794.

vanKuyk, P. A., Diderich, J. A., MacCabe, A. P., Hererro, O., Ruijter, G. J. G. \& Visser, J. (2004). Aspergillus niger $m s t A$ encodes a high affinity sugar $: \mathrm{H}^{+}$symporter which is regulated in response to extracellular pH. Biochem J 379, 375-383.

Vishniac, W. \& Santer, M. (1957). The thiobacilli. Bacteriol Rev 21, 195-213.

Walsh, M. C., Smits, H. P., Scholte, M. \& van Dam, K. (1994). Affinity of glucose transport in Saccharomyces cerevisiae is modulated during growth on glucose. J Bacteriol 176, 953-958.

Wei, H., Vienken, K., Weber, R., Bunting, S., Requena, N. \& Fischer, R. (2004). A putative high affinity hexose transporter, $h x t A$, of Aspergillus nidulans is induced in vegetative hyphae upon starvation and in ascogenous hyphae during cleistothecium formation. Fungal Genet Biol 41, 148-156.

Wieczorke, R., Krampe, S., Weierstall, T., Freidel, K., Hollenberg, C. P. \& Boles, E. (1999). Concurrent knock-out of at least 20 transporter genes is required to block uptake of hexoses in Saccharomyces cerevisiae. FEBS Lett 464, 123-128.

Edited by: B. A. Horwitz 